\title{
Efficacy and tolerability of exclusive enteral nutrition in adult patients with complicated Crohn's disease
}

\author{
Sanchit Sharma ${ }^{1}$, Arti Gupta ${ }^{1}$, Saurabh Kedia ${ }^{1}$, Samagra Agarwal $^{1}$, Namrata Singh ${ }^{1}$, Sandeep Goyal ${ }^{1}$, Saransh Jain ${ }^{1}$, \\ Vipin Gupta ${ }^{1}$, Pabitra Sahu ${ }^{1}$, Sudheer Kumar Vuyyuru ${ }^{1}$, Bhaskar Kante ${ }^{1}$, Raju Sharma ${ }^{2}$, Rajesh Panwar ${ }^{3}$, Peush Sahni ${ }^{3}$, \\ Govind Makharia ${ }^{1}$, Vineet Ahuja ${ }^{1}$ \\ Departments of ${ }^{1}$ Gastroenterology and Human Nutrition, ${ }^{2}$ Radiodiagnosis, and ${ }^{3}$ Gastrointestinal Surgery, All India Institute of Medical \\ Sciences, New Delhi, India
}

Background/Aims: Exclusive enteral nutrition (EEN), an established modality for pediatric Crohn's disease (CD) is seldomly utilized in adults. The present study reports the outcome of EEN in adult CD patients at a tertiary care hospital in India. Methods: This was a retrospective analysis of CD patients who received EEN as a sole modality/adjunct to other treatment. The primary and secondary outcomes changed in Crohn's Disease Activity Index (CDAI), and clinical response (decline in CDAI $>70$ ), respectively, at 4 and 8 weeks. Subgroup analysis evaluated response across different phenotypes, EEN formulations and prior treatment. Linear mixed effect model was created to assess the predictors of EEN response. Results: Thirty-one CD patients received EEN over median duration of 4 weeks (range, 2-6 weeks). CDAI showed a significant improvement post EEN at 4 (baseline 290 [260-320] vs. 240 [180-280], $P=0.001$ ) and 8 weeks (baseline 290 [260-320] vs. 186 [160-240], $P=0.001$ ), respectively. The cumulative clinical response rates at 4 and 8 weeks were $37.3 \%$ and $80.4 \%$ respectively. The clinical response rates at 8 weeks across B1 $(n=4)$, B2 $(n=18)$, and B3 $(n=9)$ phenotypes were $50 \%, 78.8 \%$, and $100 \%$ respectively (log-rank test, $P=0.093)$. The response rates at 8 weeks with polymeric $(\mathrm{n}=8)$ and semi-elemental diet $(\mathrm{n}=23)$ were $75 \%$ and $82.6 \%$ respectively (log-rank test, $P=0.49$ ). Baseline CDAI (odds ratio, 1.008; 95\% confidence interval, $1.002-1.017 ; P=0.046$ ) predicted response to EEN. Conclusions: EEN was effective in inducing clinical response across different phenotypes of CD. Baseline disease activity remained the most important predictor of clinical response to EEN. (Intest Res 2021;19:291-300)

Key Words: Exclusive enteral nutrition; Crohn disease; Crohn's Disease Activity Index; Inflammation

\section{INTRODUCTION}

Crohn's disease (CD) is a chronic, progressive, inflammatory disease of the bowel with manifestations involving any part of gastrointestinal (GI) tract. ${ }^{1}$ The pathogenesis of CD involves an inflammatory damage to the intestine due to an aberrant immune response against the environment, primarily the dys-

Received December 16, 2019. Revised February 28, 2020

Accepted March 21, 2020.

Correspondence to Vineet Ahuja, Department of Gastroenterology, All India

Institute of Medical Sciences, Room No. 3093, 3rd floor, Academic Building,

New Delhi 110029, India. Tel: +91-11-26593300, Fax: +91-11-26588663,

E-mail:vineet.aiims@gmail.com biotic gut microbiome, in genetically predisposed individuals. Diet, through its influence on the gut microbiome plays a major role in the pathogenesis of $\mathrm{CD}$, and studies have correlated certain dietary constituents with the risk of CD onset as well as progression. ${ }^{2-5}$ Dietary beliefs play an important role in disease perception, as many patients with CD make dietary changes which can lead to malnutrition. ${ }^{6}$ Another important role of diet is its influence on the body composition, the dynamics of which varies with disease severity and duration. ${ }^{7.8}$ The major treatment strategies in CD aim to arrest the aberrant immune response, but because of their nonspecific nature, these are limited by adverse effects, cost and limited efficacy. The adverse effects assume importance particularly with 
the ever increasing use of anti-tumor necrosis factor (TNF) agents for the management of $\mathrm{CD}$ in the current era. ${ }^{9}$ Dietary manipulation would be a more physiological and safer strategy to manage the $\mathrm{CD}$ associated inflammation, with an additional advantage of improving the nutritional status of these patients.

There are various dietary strategies which have been tested for the management of $\mathrm{CD}$, but the principle behind all these strategies involves the enrichment and depletion of anti-inflammatory and pro-inflammatory components respectively. ${ }^{3}$ Exclusive enteral nutrition (EEN) is an extreme form of dietary manipulation, defined as the use of a complete liquid diet, with the exclusion of normal dietary components for a defined period of time, as a therapeutic measure to induce remission. ${ }^{10}$ EEN in the form elemental diet, semi-elemental diet and or polymeric formula-based diet has been shown to be as effective as steroids in the management of pediatric $\mathrm{CD}^{1{ }^{11-13}}$ Because of the side effects of steroids on growth and development, EEN is the recommended treatment for induction of remission in pediatric CD. ${ }^{14,15}$ There is an emerging but limited evidence of EEN in adults. Recent studies have demonstrated its efficacy in inflammatory stricturing disease, in complicated disease like abscess, postoperative recurrence and in fistulizing variants of $\mathrm{CD}^{16-18}$ although, the randomized controlled trials performed till date have found it inferior to steroids. ${ }^{19}$ Presently, EEN is recommended only as an adjunctive treatment in adults with $\mathrm{CD}^{20}$

In view of limited evidence regarding use of EEN in adults with $\mathrm{CD}$, we undertook this study to ascribe the role of EEN in the management of $\mathrm{CD}$.

\section{METHODS}

\section{Patients}

Consecutive patients with CD who received EEN and were under follow-up at inflammatory bowel disease (IBD) clinic, All India Institute of Medical Sciences, New Delhi, India between October 2017 and May 2019 were included.

\section{Study Design}

It was a retrospective analysis of a prospectively maintained database of patients with CD who received EEN. The decision to start EEN was taken by physicians managing patients in IBD clinic, and the major indications were active disease despite being on immunosuppressive therapy, active disease and contra-indication to immunosuppressive therapy like ab- scess, active enterocutaneous fistula with features of sepsis, and as an adjunct to immunosuppressive therapy (for patients who required nutritional built up prior to administration of biologicals). Active CD was defined as Crohn's Disease Activity Index $(\mathrm{CDAI})>150,{ }^{21}$ and the following treatment regimens were utilized: corticosteroids, immunomodulators like azathioprine and methotrexate and anti-TNF- $\alpha$ agents like infliximab/adalimumab. The disease location and behavior was classified as per Montreal classification: ${ }^{22}$ B1-inflammatory disease; B2-stricturing disease, B3-fistulizing disease; L1-terminal ileal disease; L2-colonic disease; L3-ileocolonic disease. Complicated CD was defined as development of stricture, fistula or abscess or requirement of surgery in patients with $\mathrm{CD}^{23}$ Antibiotics were administered in patients who had evidence of active infection.

The following parameters were analyzed from the database of these patients: baseline clinical and demographic profile including age, sex, disease duration, and phenotype, type of treatment received prior to initiation of EEN, and laboratory parameters like hemoglobin and serum albumin, which were also assessed at 4 and 8 weeks. CDAI was assessed at baseline and at 4 and 8 weeks after starting EEN. Clinical response was defined as decline in CDAI by 70 points or more from the baseline. ${ }^{24}$ Clinical remission was defined as absolute reduction of CDAI to less than 150 post-intervention. ${ }^{24}$ All procedures performed in studies were in accordance with the ethical standards of the institutional ethics committee and in accordance with the 1975 Helsinki Declaration and its later amendments or comparable ethical standards (Ref No. IECPG-219/27.03.2019). Written informed consent was obtained from all included patients.

\section{Intervention}

The formulation used in our patients was both semi-elemental based (Peptamen; Nestle Health Science) and polymeric based (Fresubin; Fresenius Kabi India), and depended upon the availability and patient preference. The patients were instructed about the method of preparation of EEN and the calories and protein requirement were calculated according to standard formulae by the IBD dietician. The patients were also instructed about abstaining from any other food items during the duration of EEN intake. The dietician kept a track of patient intake, compliance, and tolerance to EEN, both for admitted patients (daily patient rounds) and out-patients (regular telephonic interviews and in-person interview at clinic visits). The amount of formula being given was recorded and any subse- 
quent intolerance or other side effects were noted. The route of administration was per orally or through naso-enteric tube in all patients. The patients were followed at 4 and 8 weeks or in-between in case of exacerbation of disease activity or intolerance to EEN.

\section{Outcomes}

The primary outcome assessed was improvement in CDAI post EEN at 4 and at 8 weeks. Secondary outcomes were: (1) changes in hemoglobin and serum albumin post EEN institution at 4 and at 8 weeks. (2) Proportion of patients having clinical response and remission while on EEN assessed at 4 and at 8 weeks respectively. (3) Identification of predictors of clinical response to EEN. In addition, a subgroup analysis to compare outcomes was done with respect to type of EEN, previous treatment strategies and compare when EEN was used as a sole treatment modality.

\section{Statistical Analysis}

Baseline parameters with normal distribution were expressed as mean \pm standard deviation, and those with skewed distribution were expressed using median (interquartile range). Qualitative data were presented as number (\%). Comparison of disease response parameters like CDAI, hemoglobin and serum albumin pre and post EEN intervention was made using paired $t$-test or Wilcoxon rank sum test as appropriate. A test was considered as significant if $P$-value $<0.05$. Change in CDAI over time was modelled using linear mixed effects model, keeping subject ID as random effects and time after initiating EEN as fixed effects. Significance of change over time was estimated. Profile plots of change in CDAI, hemoglobin and albumin over time was presented stratified according to disease behavior along with individual patient parameters.
Cumulative clinical response rates were calculated and represented using Kaplan-Meier plot for overall group and were also stratified according to disease behavior. The data was entered using Microsoft Excel 2011 (Microsoft Corp., Redmond, WA, USA) and was analyzed using R studio (Boston, MA, USA). In addition to the base packages in R, ggplot2, lmer, cart, dplyr, and tidyr packages were used.

\section{RESULTS}

EEN was started in 35 patients of complicated CD during the study period of October 2017 to May 2019. In view of initial intolerance within first 7 days, 4 patients discontinued EEN and 31 patients were included for final analysis (Fig. 1).

\section{Baseline Demographic and Clinical Characteristics}

The baseline characteristics of the cohort is summarized in Table 1 . Mean age of the cohort was $34 \pm 16$ years with a slight male preponderance (54.8\%). Stricturing disease (58.1\%) was the most common phenotype followed by penetrating (29.0\%) and non-stricturing, non-penetrating disease (12.9\%). Terminal ileal (38.7\%) was the most common site of involvement followed by ileocolonic (32.3\%), colonic (16.1\%) and combined ileal and upper GI tract involvement (12.9\%).

\section{Indications of EEN}

Semi-elemental based preparation of EEN was started in 23 patients $(74.2 \%)$ whereas 8 patients $(25.8 \%)$ were started on polymeric based preparation. The median duration of EEN administration was 4 weeks (interquartile range, 2-6 weeks). The most common indication of EEN was active disease despite being on immunosuppressive therapy (53.1\%) followed by active disease in the presence of contraindications for im-

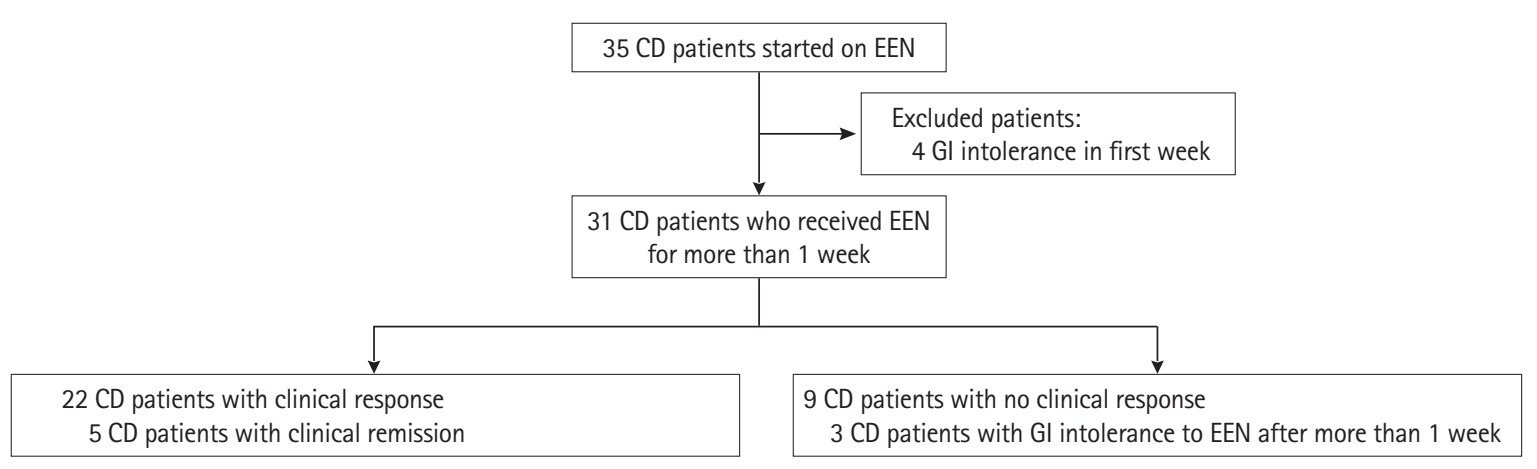

Fig. 1. Flowchart of the patient recruitment in the study. $C D$, Crohn's disease; $E E N$, exclusive enteral nutrition; $G$, gastrointestinal. 
Table 1. Baseline Demographic and Clinical Characteristics of Crohn's Disease Patients Who Received EEN

\begin{tabular}{|c|c|}
\hline Variable & Cohort $(n=31)$ \\
\hline Age (yr) & $34 \pm 16$ \\
\hline Sex, male/female & $17(54.8) / 14(45.2)$ \\
\hline Duration of symptoms (mo) & $48(18-72)$ \\
\hline B1 (inflammatory) & $4(12.9)$ \\
\hline B2 (stricturing) & $18(58.1)$ \\
\hline Inflammatory stricture & $8 / 18(44.4)$ \\
\hline Fibrotic stricture & 10/18 (55.6) \\
\hline B3 (penetrating) & $9(29.0)$ \\
\hline L1 (terminal ileal+cecal) & $12(38.7)$ \\
\hline L2 (colonic) & $5(16.1)$ \\
\hline L3 (ileocolonic) & $10(32.3)$ \\
\hline L4 (proximal ileal and above) & $4(12.9)$ \\
\hline Perianal disease & $5(16.1)$ \\
\hline \multicolumn{2}{|l|}{ Previous treatment received } \\
\hline Corticosteroid & $30(96.7)$ \\
\hline Azathioprine & $22(70.9)$ \\
\hline Methotrexate & $6(19.3)$ \\
\hline Anti-TNF- $\alpha$ agents & $5(16.1)$ \\
\hline Surgery & $13(41.9)$ \\
\hline \multicolumn{2}{|l|}{ Baseline investigations } \\
\hline Hemoglobin (g/dL) & $7.7(6.5-8.0)$ \\
\hline Albumin (g/dL) & $2.5(1.8-3.0)$ \\
\hline Baseline CDAI & $290(260-320)$ \\
\hline \multicolumn{2}{|l|}{ Current treatment } \\
\hline Steroids+EEN & $6(19.3)$ \\
\hline Azathioprine+EEN & $2(6.5)$ \\
\hline Methotrexate+EEN & $1(3.2)$ \\
\hline Anti-TNF- $\alpha$ therapy+EEN & $11(35.5)$ \\
\hline EEN alone & $11(35.5)$ \\
\hline \multicolumn{2}{|l|}{ Type of EEN } \\
\hline Semi-elemental & $23(74.2)$ \\
\hline Polymeric & $8(25.8)$ \\
\hline Duration of EEN (wk) & $4(2-6)$ \\
\hline
\end{tabular}

Values are presented as mean \pm standard deviation, number (\%), or median (interquartile range).

EEN, exclusive enteral nutrition; CDAl, Crohn's Disease Activity Index; TNF, tumor necrosis factor.
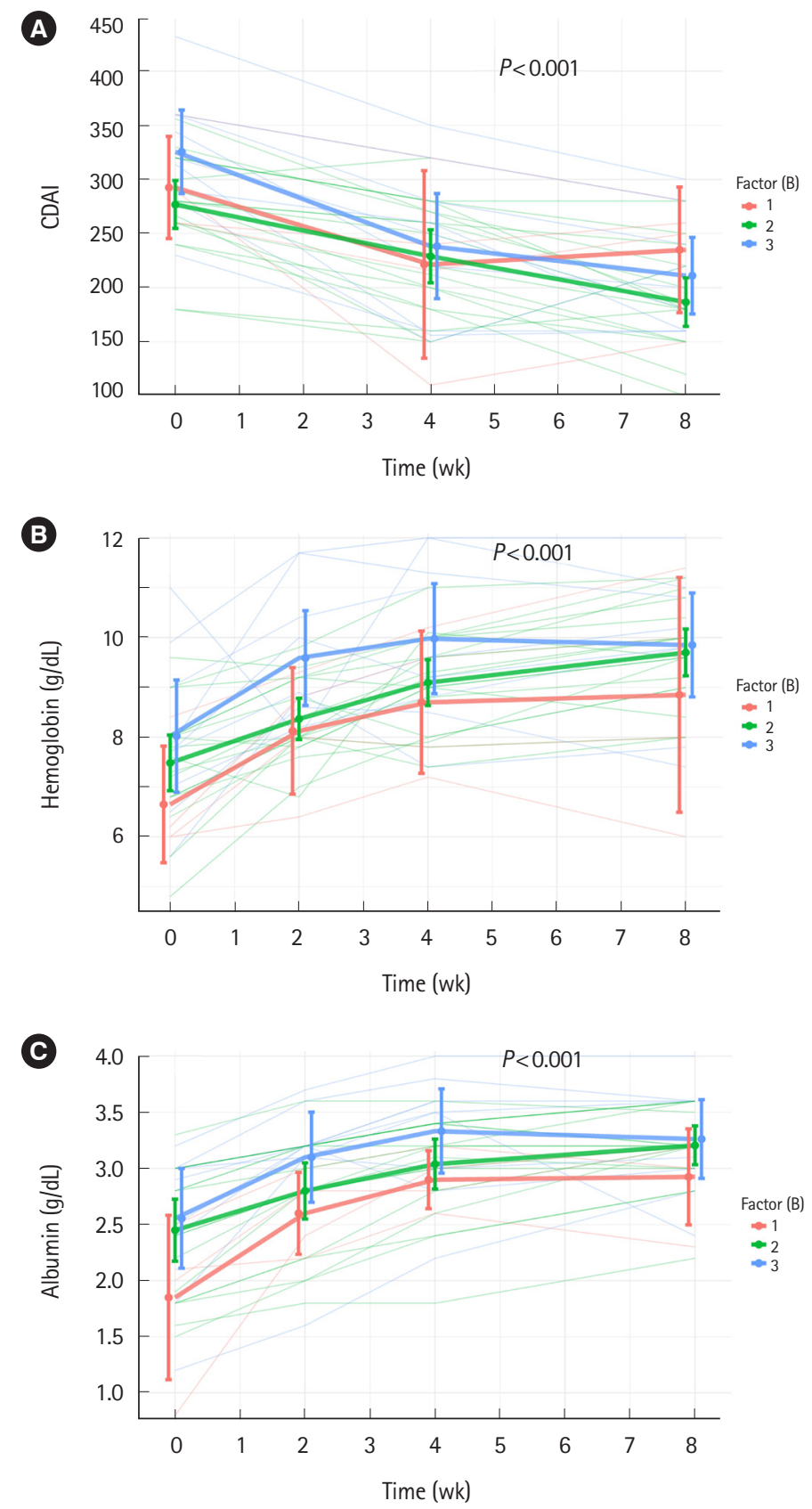

Fig. 2. Profile plots depicting change in Crohn's Disease Activity Index (CDAl) (A), hemoglobin (B), and serum albumin (C) levels with exclusive enteral nutrition administration across different phenotypes of Crohn's disease assessed at 4 and 8 weeks respectively.

Table 2. Change in CDAl, Hemoglobin, and Serum Albumin Levels Assessed at 4 and 8 Weeks Post EEN

\begin{tabular}{|c|c|c|c|c|c|}
\hline Variable & Baseline & 4 Weeks (post EEN) & $P$-value & 8 Weeks (post EEN) & $P$-value \\
\hline CDAl & $290(260-320)$ & $240(180-280)$ & 0.001 & $186(160-240)$ & 0.001 \\
\hline Hemoglobin (g/dL) & $7.7(6.5-8.0)$ & $9.2(8.0-10.0)$ & 0.001 & $9.8(9.0-10.8)$ & 0.001 \\
\hline
\end{tabular}

Values are presented as median (interquartile range).

CDAl, Crohn's Disease Activity Index; EEN, exclusive enteral nutrition. 
munosuppression (34.3\%). Other patients were given EEN for build-up of nutrition in patients with active disease who required immunosuppression. EEN was started as an adjunct to steroids (19.3\%), immunomodulators (9.7\%), anti-TNF- $\alpha$ therapy $(35.5 \%)$ while it was used as the only modality of treatment in $35.5 \%$ of patients.

\section{Changes in Parameters Post EEN}

The changes in parameters post EEN administration are summarized in Table 2 . The primary outcome assessed by change in CDAI showed a significant improvement post administration of EEN at 4 weeks (baseline 290 [260-320] vs. 240 [180280], $P=0.001$ ) and at 8 weeks (baseline 290 [260-320] vs. 186 [160-240], $P=0.001$ ) respectively (Fig. 2A). Hemoglobin showed a significant rise at 4 weeks (baseline 7.7 [6.5-8.0] g/dL vs. 9.2 $[8.0-10.0] \mathrm{g} / \mathrm{dL}, P=0.001$ ) and at 8 weeks (baseline $7.7[6.5-$ 8.0] g/dL vs. 9.8 [9.0-10.8] g/dL, $P=0.001$ ) (Table 2, Fig. 2B). Serum albumin also showed a significant improvement post administration of EEN at 4 weeks (baseline 2.5 [1.8-3.0] g/dL vs. $3.2[2.8-3.4] \mathrm{g} / \mathrm{dL}, P=0.001$ ) and at 8 weeks (baseline 2.5 [1.8-3.0] g/dL vs. 3.2 [3.0-3.6] g/dL, $P=0.001$ ) (Fig. 2C).

\section{Response to EEN in Whole Cohort and in Subgroups}

Clinical response $(\mathrm{CDAI}$ reduction $>70)$ to $\mathrm{EEN}$ was seen in 22 out of 31 patients at 8 weeks in the whole cohort $(70.9 \%)$. The cumulative clinical response rates at 4 and 8 weeks were $37.3 \%$ and $80.4 \%$ respectively (Fig. 3 ). Five out of 31 patients $(16.1 \%)$ achieved clinical remission $(\mathrm{CDAI}<150)$ at 8 weeks.
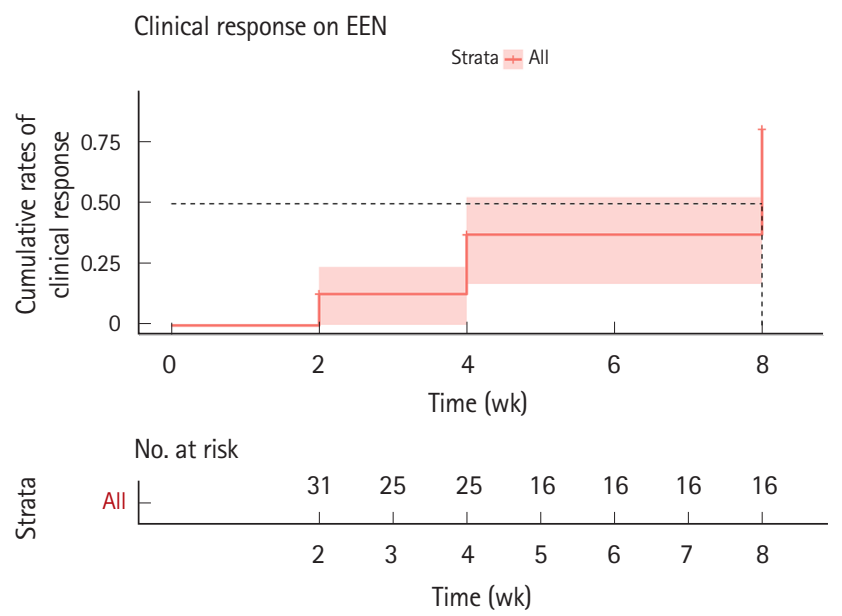

Fig. 3. Kaplan-Meier response plot showing clinical response to exclusive enteral nutrition (EEN). The cumulative clinical response rates to EEN at 4 and 8 weeks were $37.3 \%$ and $80.4 \%$ respectively.
Among different phenotypes, 1 patient of B1 disease (25\%), 4 patients of B2 disease (27.7\%) and none of the patients in B3 disease could achieve clinical remission.

The efficacy of EEN across different subgroups and treatment arms was analyzed. The clinical response rates at 8 weeks across B1, B2, and B3 disease were 50\%, 78.8\%, and 100\% respectively (log-rank test, $P=0.093$ ) (Fig. 4). EEN when used as the only treatment for CD $(\mathrm{n}=11)$, showed significant improvement in CDAI at 4 weeks (280 [260-320] vs. 220 [180-240], $P<0.050)$ and at 8 weeks (190 [170-220], $P=0.050)$ (Supplementary Fig. 1). Clinical response was present in 7 patients (63.6\%) while clinical remission was seen in 1 patient who were only on EEN without concurrent pharmacological therapy. The response rates at 8 weeks with polymeric and semi-elemental diet were $75 \%$ and $82.6 \%$ respectively (log-rank test, $P=0.49$ ) (Supplementary Fig. 2). In the subgroup analysis comparing different arms of treatment received, the response rates at 8 weeks in patients who previously received immunomodulators and anti-TNF therapy were $59 \%$ and $80 \%$ respectively.

\section{Predictors of Clinical Response on EEN}

A linear mixed effect model was created using variables like type of EEN, phenotype of disease, disease duration, disease location, baseline CDAI, baseline serum albumin and hemoglobin levels and EEN used alone/adjunct to other treatment modalities to analyze which factor remains predictive of clinical response with EEN in CD. In this model, on univariate analysis, only baseline CDAI (odds ratio, 1.008; 95\% confidence in-

Clinical response on EEN

Strata $+B=1+B=2+B=3$
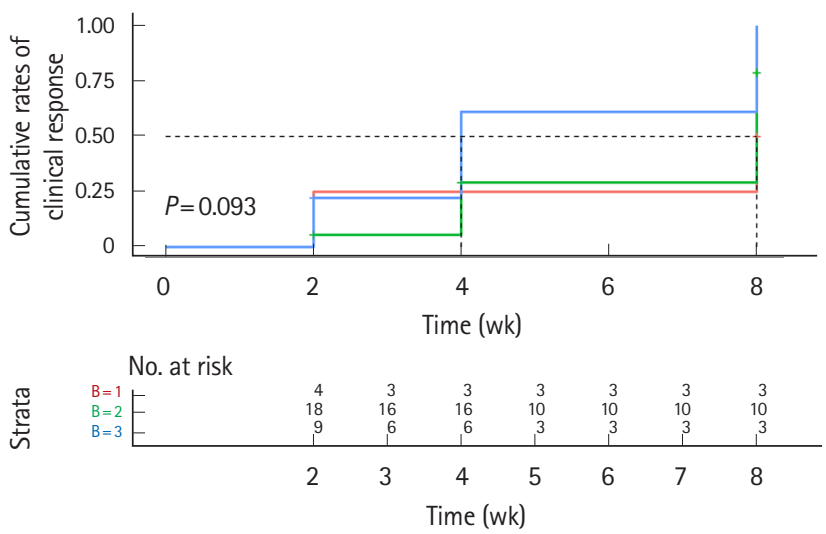

Fig. 4. Kaplan-Meier response plot showing clinical response across different phenotypes of disease. The clinical response rates at 8 weeks in B1 $(n=4), B 2(n=18)$, and B3 $(n=9)$ disease were $50 \%, 78.8 \%$, and 100\% respectively (log-rank test, $P=0.093$ ). 
Table 3. Univariate Model Incorporating Different Variables for Predicting Clinical Response to EEN in CD Patients

\begin{tabular}{lcc}
\hline Variable & $\begin{array}{c}\text { OR }(95 \% \mathrm{Cl}) \text { for CDAI } \\
\text { response }\end{array}$ & $P$-value \\
\hline $\begin{array}{l}\text { Disease duration } \\
\text { Phenotype of disease }\end{array}$ & $1.000(0.983-1.017)$ & 0.983 \\
B1 vs. B2 & $1.880(0.414-8.474)$ & 0.411 \\
B1 vs. B3 & $4.345(0.893-21.12)$ & 0.068 \\
Site of disease & $1.409(0.309-6.398)$ & 0.659 \\
L1 vs. L2 & $0.723(0.211-2.475)$ & 0.605 \\
L1 vs. L3 & $1.008(1.002-1.017)$ & 0.046 \\
CDAl (baseline) & $1.046(0.840-1.564)$ & 0.389 \\
Hemoglobin & $1.369(0.652-2.387)$ & 0.406 \\
Serum albumin & & \\
Type of EEN & $1.050(0.138-7.986)$ & 0.962 \\
$\quad$ Semi-elemental & $1.782(0.093-6.522)$ & 0.821 \\
\hline
\end{tabular}

Behavior of disease described according to Montreal classification: B1inflammatory disease; B2-stricturing disease; B3-fistulizing disease; L1-terminal ileal disease; L2-colonic disease; L3-ileocolonic disease. Multivariate analysis not presented as only one factor was significant on univariate analysis.

EEN, exclusive enteral nutrition; $C D$, Crohn's disease; $\mathrm{OR}$, odds ratio; $\mathrm{Cl}$, confidence interval; CDAl, Crohn's Disease Activity Index.

terval, $1.002-1.017 ; P=0.046$ ) could predict clinical response while all other variables were non-significant (Table 3).

\section{Adverse Events}

None of the patients developed serious adverse events. Overall, GI intolerance was the most common adverse event seen in 7 out of 35 patients (20\%) (initial cohort) (Fig. 1). Of these, 4 patients discontinued EEN within 1st week and 3 additional patients stopped EEN after a median duration of 10 days. All these patients were given semi-elemental based preparation of EEN.

\section{DISCUSSION}

EEN as a therapy for CD was serendipitously discovered in 1973 by Voitk et al. ${ }^{25}$ who observed that the patients given EEN for preoperative build up improved clinically and many could avoid surgery. This dual ability of EEN in inducing the clinical response as well as a supporting tool for nutritional built-up, makes it an important therapeutic option both as an adjunct or exclusively in the management of $\mathrm{CD}$. This is especially true in the settings where other treatment options may be contra- indicated (as in active infection with active disease) and in malnourished patients. EEN is already the first line therapy for induction of remission in pediatric $\mathrm{CD}^{14}$ and has even demonstrated better efficacy than steroids in terms of improvements in growth and mucosal healing. ${ }^{26,27}$ The evidence for adults is heterogeneous, with meta-analyses reporting it as inferior to steroids ${ }^{28}$ and recent case series demonstrating improvement in complicated $\mathrm{CD}^{29}$ The treatment options for complicated $\mathrm{CD}$ in the developing countries become limited by the higher risks of infectious adverse events, especially when these patients are malnourished. The evidence and awareness for EEN in this setting is scarce, and we have found good results with the use of EEN in our patients for the past 18 months.

In the present study, there was a significant improvement in clinical activity and biochemical parameters post administration of EEN in patients with CD, most of whom had a complicated phenotype. More than $80 \%$ patients had either stricturing or fistulizing disease, $>90 \%$ were steroid experienced, $16.1 \%$ patients had received anti-TNF and $41.9 \%$ had undergone CD specific surgery in the past. Even in this cohort of patients, more than two-thirds experienced clinical response at 8 weeks. EEN was effective both as an adjunct and as well as the only treatment in inducing remission. Baseline disease activity was the most important factor predicting clinical response in these patients.

The results of the present study are in line with the recent studies evaluating the role of EEN in adults with CD. Wall et al. ${ }^{18}$ in their pilot study found 2 weeks of EEN was effective in inducing clinical remission in adults with CD. Interestingly, the authors of this study demonstrated further treatment with either partial or EEN had comparable treatment outcomes. However, this study had a predominantly B1 disease (78.9\%), unlike the present study, which could account for better outcomes even at 2 weeks. Moreover, the present study had a sicker subgroup of patients with $93 \%$ and $40 \%$ patients having received corticosteroids and anti-TNF agents, respectively, while in the study from Wall et al, no patient had received steroids and only 5.2\% patients had received biologicals. In addition, duration of disease also differed with patients in our study having a longer 48 months (range, 18-72 months) disease duration compared with median of 7 months in this study.

Hu et al. ${ }^{30}$ in their study of 59 adult CD patients with inflammatory strictures found EEN to be effective in inducing clinical remission in $64.6 \%$ of patients when given over 12 weeks, which was higher when compared with our study, where though the response rate was $78.8 \%$, clinical remission was seen 
only in $27.7 \%$ in stricturing disease. Inclusion of only inflammatory strictures and longer duration of EEN administration (12 weeks) in this study could account for this difference in clinical remission. In the present study, patients with both inflammatory (44.4\%) and fibrotic strictures (55.6\%) were included and EEN was administered for median duration of 4 weeks (range, 2-6 weeks).

Enteral nutrition has also been used as an adjunct to infliximab in fistulizing CD. Wu et al. ${ }^{31}$ found that infliximab in combination with EEN was more effective in management of fistulizing CD when compared with infliximab alone. In the present study, of the 9 patients with fistulizing $\mathrm{CD}$, although none could achieve clinical remission (CDAI $<150$ ), clinical response rates to EEN were $100 \%$. The response rates did not differ when EEN was used exclusively or as an adjunct to anti-TNF agents.

EEN has also been found to be useful when used in patients who have lost response to anti-TNF, as evidenced by a recent study which demonstrated usefulness of EEN in patients on anti-TNF agents with loss of response (LOR). ${ }^{32}$ In the present study, out of 5 patients who had LOR on anti-TNF, 4 patients $(80 \%)$ had clinical response $(\Delta \mathrm{CDAI}>70)$ at 8 weeks post EEN administration. A recent meta-analysis also supported the use of enteral nutrition to maintain response to anti-TNF molecules. ${ }^{33}$ Restoration of increased intestinal permeability and decline in mucosal production of TNF- $\alpha$ could be one of the possible explanations for benefit of EEN in patients who had LOR to anti-TNF agents. ${ }^{34}$

Importantly, the clinical response and remission rates with EEN were similar across different phenotypes of disease and could improve disease activity as the only treatment in patients who could not be started on any standard treatment of CD in view of its contraindications. Previous studies had shown different results about the efficacy of EEN in different subtypes of CD. Afzal et al. ${ }^{35}$ in their study of 65 patients with pediatric $\mathrm{CD}$ found colonic disease to be less responsive than ileocolonic and isolated ileal disease, whereas Buchanan et al. ${ }^{36}$ in their study of 110 patients found that $80 \%$ of their patients achieved remission irrespective of site of disease.

There is an emerging yet unclear role of EEN in the management of adults with CD. A systematic review showed EEN to be inferior to steroids in inducing remission in adults with $\mathrm{CD}{ }^{19}$ A Cochrane review evaluating the overall impact of EEN and its comparison with steroids found steroids to be more beneficial when compared with EEN in adults. ${ }^{28}$ However, the overall quality of evidence from the above conducted systematic reviews is low with significant heterogeneities among the stud- ies that were analyzed. Prospective uncontrolled trials have shown benefit of EEN in adults across various subtypes of disease, in the presence of complicated disease (abscess) and reduction in postoperative recurrence/complications. ${ }^{16-18}$ In the absence of well controlled randomized trials, the current guidelines do not recommend EEN in adults patients with $\mathrm{CD}^{20}$ and highlights the need of further studies in this aspect.

One of the major limitations of EEN is its GI intolerance (diarrhea, bloating, and flatulence) and unpalatability. The compliance to EEN especially in semi-elemental preparation often limits its clinical effectiveness. Overall, 7 patients (20\%) had intolerance in whom EEN had to be discontinued. All these patients were on semi-elemental preparation. Another important limitation of EEN is its cost. The average daily cost of semielemental based preparation and polymeric based preparation in India is Rs 1,200 (17 USD) and Rs 800 (11 USD) respectively. We could not perform a formal cost-effective analysis comparing different treatment options.

Our study had few limitations. First, is its retrospective design with a small sample size. Although we retrieved all data from a prospectively maintained database for IBD-CD, it is possible that we might have missed capturing some data. The sample size of the present study was small. However, EEN in adults with CD is a relatively newer concept which is still evolving. This study is the first to report the outcomes of EEN in CD from this region. Further, all the consecutive patients started on EEN were included in the study. Secondly, the criteria for activity assessment in CD was clinical using CDAI. The endoscopic disease activity and biochemical parameters like fecal calprotectin were not assessed in this study. Thirdly, the limited duration of EEN administration and a limited duration of follow-up. The limited number of patients showing clinical remission could have been because of the shorter duration of EEN administration as compared to previous studies. It is not known whether the clinical improvement seen post EEN could be sustained post discontinuation of the therapy. Fourth, EEN as the only modality of treatment was used in only $35.5 \%$ patients. Thus, the results of efficacy for EEN could have been affected by response to other medicines. However, as mentioned clinical response seen in EEN alone patients was comparable to those of the whole cohort. Further studies using a larger sample size are required to test the efficacy of EEN alone in management of adults with CD. Fifth, the improvement in the disease activity in patients with $\mathrm{CD}$ with active infections could have been because of antibiotics which were administered with EEN. However, for disease complicated with infections, 
evaluating the efficacy of EEN alone in this setting might be difficult as administration of antibiotics in this setting is the standard of care. Previous studies demonstrating effectiveness of EEN in this setting had also administered antibiotics. ${ }^{29,37}$ Sixth, the benefit sustained with EEN might reflect the changes in nutritional status before and after treatment evaluated. As seen in pediatric $\mathrm{CD}$, the effect of EEN might be due to both improvement in disease activity and improvement in nutritional status. The efficacy of EEN in this setting can be conclusively demonstrated only in the presence of a controlled group given a calorie and protein matched normal diet. This remains a major limitation of our study. Further, prospective studies with a larger sample size are required to validate these findings and to address these limitations.

To conclude, EEN is an effective therapeutic option for patients with complicated CD, especially when they are malnourished and have contraindications which preclude the use of immunosuppressive therapy. EEN is effective both as an adjunct and as only therapy, across all subtypes of $\mathrm{CD}$, and the baseline disease activity remains the most important predictor of response to therapy.

\section{ADDITIONAL INFORMATION}

\section{Funding Source}

The authors received no financial support for the research, authorship, and/or publication of this article.

\section{Conflict of Interest}

No potential conflict of interest relevant to this article was reported.

\section{Author Contribution}

Conceptualization: Ahuja V. Methodology: Ahuja V, Kedia S. Formal analysis: Sharma S, Agarwal S. Project administration: Ahuja V. Visualization: Ahuja V, Sharma S, Kedia S. Writing -original draft: Sharma S, Agarwal S, Gupta A, Singh N, Vuyyuru SK, Kante B, Sahu P, Makharia G. Writing -review and editing: Ahuja V, Kedia S, Sharma S, Sharma R, Panwar R, Sahni P, Goyal S, Jain S, Gupta V. Approval of final manuscript: all authors.

\section{ORCID}

Sharma S

Gupta A

Kedia S
Agarwal S

Singh N

Goyal S

Jain S

Gupta V

Sahu P

Vuyyuru SK

Kante B

Sharma R

Panwar R

Sahni P

Makharia G

Ahuja V https://orcid.org/0000-0002-2053-2069

https://orcid.org/0000-0002-8912-2949

https://orcid.org/0000-0003-2890-9593

https://orcid.org/0000-0002-2947-7836

https://orcid.org/0000-0002-9620-1696

https://orcid.org/0000-0002-9847-0136

https://orcid.org/0000-0002-8281-3237

https://orcid.org/0000-0001-5363-7273

https://orcid.org/0000-0001-5181-263X

https://orcid.org/0000-0002-8751-9766

https://orcid.org/0000-0002-6910-062X

https://orcid.org/0000-0002-2474-2194

https://orcid.org/0000-0002-1577-0118

\section{Supplementary Material}

Supplementary materials are available at the Intestinal Research website (https://www.irjournal.org).

\section{REFERENCES}

1. Torres J, Mehandru S, Colombel JF, Peyrin-Biroulet L. Crohn's disease. Lancet 2017;389:1741-1755.

2. Amre DK, D'Souza S, Morgan K, et al. Imbalances in dietary consumption of fatty acids, vegetables, and fruits are associated with risk for Crohn's disease in children. Am J Gastroenterol 2007;102:2016-2025.

3. Lee D, Albenberg L, Compher C, et al. Diet in the pathogenesis and treatment of inflammatory bowel diseases. Gastroenterology 2015;148:1087-1106.

4. Knight-Sepulveda K, Kais S, Santaolalla R, Abreu MT. Diet and inflammatory bowel disease. Gastroenterol Hepatol (N Y) 2015;11:511-520

5. Sugihara K, Morhardt TL, Kamada N. The role of dietary nutrients in inflammatory bowel disease. Front Immunol 2019;9: 3183.

6. Tomar SK, Kedia S, Upadhyay AD, et al. Impact of dietary beliefs and practices on patients with inflammatory bowel disease: an observational study from India. JGH Open 2017;1:1521.

7. Yadav DP, Kedia S, Madhusudhan KS, et al. Body composition in Crohn's disease and ulcerative colitis: correlation with disease severity and duration. Can J Gastroenterol Hepatol 2017; 2017:1215035.

8. Halkjaer J, Tjønneland A, Thomsen BL, Overvad K, Sørensen TI. Intake of macronutrients as predictors of 5-y changes in waist circumference. Am J Clin Nutr 2006;84:789-797. 
9. Agarwal A, Kedia S, Jain S, et al. High risk of tuberculosis during infliximab therapy despite tuberculosis screening in inflammatory bowel disease patients in India. Intest Res 2018; 16:588-598.

10. Day AS, Lopez RN. Exclusive enteral nutrition in children with Crohn's disease. World J Gastroenterol 2015;21:6809-6816.

11. Swaminath A, Feathers A, Ananthakrishnan AN, Falzon L, Li Ferry S. Systematic review with meta-analysis: enteral nutrition therapy for the induction of remission in paediatric Crohn's disease. Aliment Pharmacol Ther 2017;46:645-656.

12. Cohen-Dolev N, Sladek M, Hussey S, et al. Differences in outcomes over time with exclusive enteral nutrition compared with steroids in children with mild to moderate Crohn's disease: results from the GROWTH CD study. J Crohns Colitis 2018;12:306-312.

13. Connors J, Basseri S, Grant A, et al. Exclusive enteral nutrition therapy in paediatric Crohn's disease results in long-term avoidance of corticosteroids: results of a propensity-score matched cohort analysis. J Crohns Colitis 2017;11:1063-1070.

14. Ruemmele FM, Veres G, Kolho KL, et al. Consensus guidelines of ECCO/ESPGHAN on the medical management of pediatric Crohn's disease. J Crohns Colitis 2014;8:1179-1207.

15. Forbes A, Escher J, Hébuterne X, et al. ESPEN guideline: clinical nutrition in inflammatory bowel disease. Clin Nutr 2017; 36:321-347.

16. Adamji M, Day AS. An overview of the role of exclusive enteral nutrition for complicated Crohn's disease. Intest Res 2019;17: 171-176.

17. Li Y, Zuo L, Zhu W, et al. Role of exclusive enteral nutrition in the preoperative optimization of patients with Crohn's disease following immunosuppressive therapy. Medicine (Baltimore) 2015;94:e478.

18. Wall CL, Gearry RB, Day AS. Treatment of active Crohn's disease with exclusive and partial enteral nutrition: a pilot study in adults. Inflamm Intest Dis 2018;2:219-227.

19. Wall CL, Day AS, Gearry RB. Use of exclusive enteral nutrition in adults with Crohn's disease: a review. World J Gastroenterol 2013;19:7652-7660.

20. Gomollón F, Dignass A, Annese V, et al. 3rd European evidencebased consensus on the diagnosis and management of Crohn's disease 2016. Part 1: diagnosis and medical management. J Crohns Colitis 2017;11:3-25.

21. Best WR, Becktel JM, Singleton JW, Kern F Jr. Development of a Crohn's disease activity index. National Cooperative Crohn's Disease Study. Gastroenterology 1976;70:439-444.

22. Satsangi J, Silverberg MS, Vermeire S, Colombel JF. The Mon- treal classification of inflammatory bowel disease: controversies, consensus, and implications. Gut 2006;55:749-753.

23. Peyrin-Biroulet L, Loftus EV Jr, Colombel JF, Sandborn WJ. The natural history of adult Crohn's disease in population-based cohorts. Am J Gastroenterol 2010;105:289-297.

24. Sandborn WJ, Feagan BG, Hanauer SB, et al. A review of activity indices and efficacy endpoints for clinical trials of medical therapy in adults with Crohn's disease. Gastroenterology 2002; 122:512-530

25. Voitk AJ, Echave V, Feller JH, Brown RA, Gurd FN. Experience with elemental diet in the treatment of inflammatory bowel disease. Is this primary therapy? Arch Surg 1973;107:329-333.

26. Borrelli O, Cordischi L, Cirulli M, et al. Polymeric diet alone versus corticosteroids in the treatment of active pediatric Crohn's disease: a randomized controlled open-label trial. Clin Gastroenterol Hepatol 2006;4:744-753.

27. Yu Y, Chen KC, Chen J. Exclusive enteral nutrition versus corticosteroids for treatment of pediatric Crohn's disease: a metaanalysis. World J Pediatr 2019;15:26-36.

28. Narula N, Dhillon A, Zhang D, Sherlock ME, Tondeur M, Zachos M. Enteral nutritional therapy for induction of remission in Crohn's disease. Cochrane Database Syst Rev 2018;4:CD000542.

29. Yang Q, Gao X, Chen H, et al. Efficacy of exclusive enteral nutrition in complicated Crohn's disease. Scand J Gastroenterol 2017;52:995-1001.

30. Hu D, Ren J, Wang G, et al. Exclusive enteral nutritional therapy can relieve inflammatory bowel stricture in Crohn's disease. J Clin Gastroenterol 2014;48:790-795.

31. Wu XL, Chen RP, Tao LP, Wu JS, Chen XR, Chen WC. Infliximab combined with enteral nutrition for managing Crohn's disease complicated with intestinal fistulas. Gastroenterol Res Pract 2016;2016:5947926.

32. Hisamatsu T, Kunisaki R, Nakamura S, et al. Effect of elemental diet combined with infliximab dose escalation in patients with Crohn's disease with loss of response to infliximab: CERISIER trial. Intest Res 2018;16:494-498.

33. Hirai F, Takeda T, Takada Y, et al. Efficacy of enteral nutrition in patients with Crohn's disease on maintenance anti-TNF-alpha antibody therapy: a meta-analysis. J Gastroenterol 2020; 55:133-141.

34. Yamamoto T, Nakahigashi M, Umegae S, Kitagawa T, Matsumoto K. Impact of elemental diet on mucosal inflammation in patients with active Crohn's disease: cytokine production and endoscopic and histological findings. Inflamm Bowel Dis 2005;11:580-588.

35. Afzal NA, Davies S, Paintin M, et al. Colonic Crohn's disease in 
children does not respond well to treatment with enteral nutrition if the ileum is not involved. Dig Dis Sci 2005;50:14711475.

36. Buchanan E, Gaunt WW, Cardigan T, Garrick V, McGrogan P, Russell RK. The use of exclusive enteral nutrition for induction of remission in children with Crohn's disease demonstrates that disease phenotype does not influence clinical remission. Aliment Pharmacol Ther 2009;30:501-507.

37. Liu J, Andrews JM, Sammour T, et al. Benefits of exclusive enteral nutrition in adults with complex active Crohn's disease: a case series of 13 consecutive patients. Crohns Colitis 360 2019; 1:otz044. 
See "Efficacy and tolerability of exclusive enteral nutrition in adult patients with complicated Crohn's disease " on page 291-300.

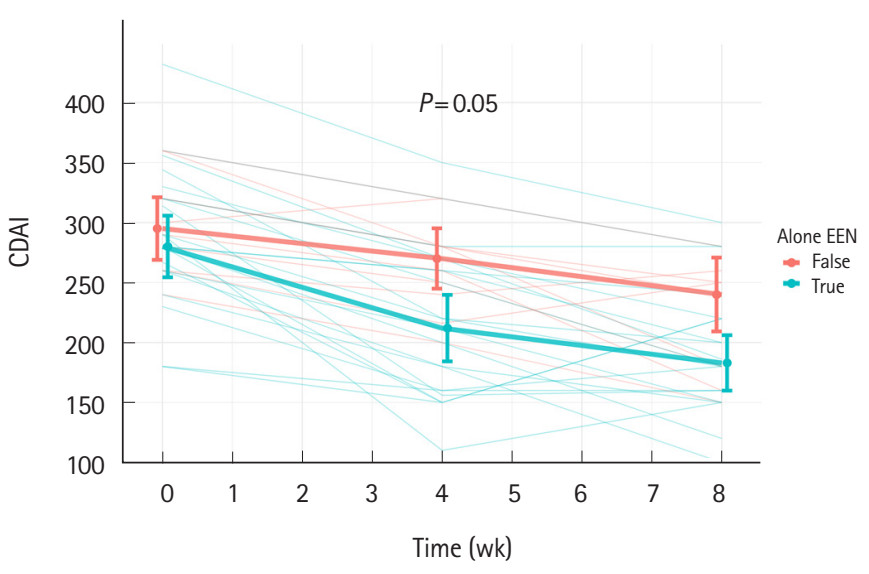

Supplementary Fig. 1. Profile plots depicting change in CDAI across subgroups when exclusive enteral nutrition (EEN) was administered as a sole modality of treatment and when it was used in combination with other treatment. There was a significant change in CDAl across both groups $(P<0.05)$.

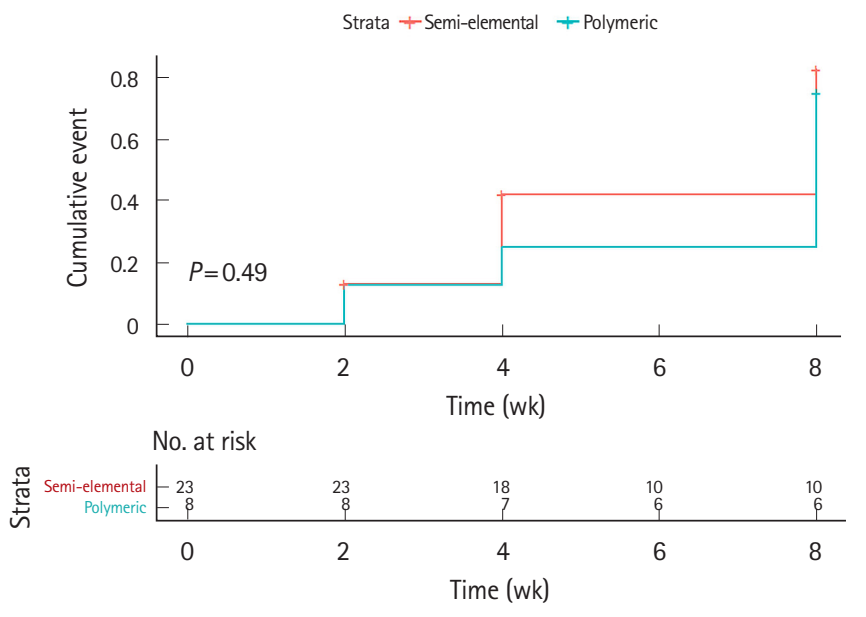

Supplementary Fig. 2. Kaplan-Meier response plot showing clinical response across different formulations of exclusive enteral nutrition. The response rates with semi-elemental $(n=23)$ and polymeric $(n=8)$ preparation were $75 \%$ and $82.6 \%$ respectively (logrank test, $P=0.49$ ). 